\title{
DESIGN CONSIDERATIONS FOR THE CESR-c WIGGLER MAGNETS *
}

\author{
J. A. Crittenden ${ }^{\dagger}$, A. Mikhailichenko, A. Temnykh \\ Laboratory for Elementary-Particle Physics, Cornell University, Ithaca, NY 14853-8001
}

\begin{abstract}
The damping-dominated beam dynamics of the CESR storage ring modified to operate in the $1.5-3 \mathrm{GeV}$ energy range impose unique and stringent design specifications on the 1.3-m-long superconducting wiggler magnets which provide the necessary damping. A superferric 7pole wiggler magnet which meets these design specifications has been designed, built and tested in situ. Studies of finite-element models and particle-tracking simulations have shown that that an 8-pole model of similar design will provide improved consistency in transfer functions when operated at peak fields which differ from the primary design value.
\end{abstract}

\section{INTRODUCTION}

The $e^{+} e^{-}$collider CESR at Cornell University is presently undergoing an upgrade which will permit studies of charmed hadron decays with unprecedented statistical accuracy [1]. The requisite luminosity at the beam energy of $1.5-3 \mathrm{GeV}$ relies on control of the emittance and on the reduction of damping time afforded by the introduction of superconducting wiggler magnets with peak fields reaching 2.1 Tesla. Over the past several years, the CESR operations group has undertaken extensive engineering studies $[2,3]$. During the past year, six wiggler magnets have been built and tested $[4,5]$. One wiggler has been installed in CESR, and a beam-based characterization of its properties has been carried out [6]. Lattice designs based on symplectic particle-tracking algorithms [7] have been developed. We report here on the accuracy obtained with finite-element-based field calculation algorithms, comparing the results to field measurements. We also discuss wiggler design considerations in relation to calculated transfer functions.

\section{FIELD CALCULATIONS}

The wiggler magnets are $1.3 \mathrm{~m}$ long, $23.8 \mathrm{~cm}$ wide, with a vertical gap of $7.62 \mathrm{~cm}$ which accommodates a beam pipe with horizontal and vertical apertures of 9 and $5 \mathrm{~cm}$. The horizontal uniformity of $2 \times 10^{-3}$ in the vertical field component over $\pm 4 \mathrm{~cm}$ is obtained via 6 -cm-wide rectangular cutouts in the pole faces with depths ranging from $3.5 \mathrm{~mm}$ to $5.5 \mathrm{~mm}$ depending on the pole length and its field strength. Two 7-pole wigglers with slightly different coil types and four 8-pole wigglers have been built and tested. Detailed information on the three wiggler designs is given in Table 1 . The longitudinally symmetric field of the 7-pole

\footnotetext{
${ }^{*}$ Work supported by the National Science Foundation

† critten@1ns.cornell.edu
}

model uses the two $15-\mathrm{cm}-$ long end poles to cancel the kick of the 20-cm-long central pole, while the asymmetry of the 8-pole design ensures zero kick to within construction tolerances. Trim coils in the end poles allow adjustment of the first and second field integrals. The wiggler field calculations were performed using the MERMAID 3D package and the magnetostatics portion of the OPERA (version 8.5 size 3) [8] software package. Exploiting the three-fold symmetry of the ideal geometry, 1/8 models were used for the highest accuracy fields. Full 3-dimensional models were used in the study of geometrical construction errors. The finite-element mesh size was limited to a maximum of $1 \mathrm{~cm}$ the magnet steel. The more detailed permeability data available in MERMAID for 1010 steel was ported to the OPERA software for these calculations. During the model development stage of the project, contributions to the field from the magnetized iron were calculated via finite-element interpolation, while the current contributions were obtained from integrals over the current elements. The final accuracy of the calculation was obtained by using the integral method for each contribution. Each of the two contributions makes up about half of the peak field in these superferric magnets.

\section{FIELD MEASUREMENTS}

Detailed field map measurements providing information on field uniformity at a level better than $10 \mathrm{G}$ for the vertical field component were obtained using a motor-driven Hall probe. Flip-coil measurements [5] were used to obtain accurate information on the horizontal and vertical field components integrated along straight lines over the length of the wiggler. The integrals of the vertical field component for several excitation levels for the second of the two 7pole wiggler magnets are shown in Fig. 1. Figure 2 shows these integrals for the first of the four 8-pole wigglers. The greater dependence on excitation exhibited by the 7 pole design results from the fact that the end pole fields compensate the central pole field. Since the peak field in the end poles is smaller, saturation effects result in such a dependence. The asymmetric field of the 8-pole design avoids this dependence. However, since a single current source is used for the end-pole trim coils in both models, this first integral cannot be controlled with the trim current in the 8-pole design, and depends critically on construction tolerances. The value of the first integral for all four of the 8-pole wigglers was measured to be less than $3 \mathrm{G}-\mathrm{m}$. The 8-pole design employs the trim coils to adjust the second integral, which is related to beam displacement. The trim current in the 7-pole design can be used to minimize the first integral and the kick. Construction tolerances then 
Table 1: CESR-c wiggler geometrical parameters and operating currents for $2.1 \mathrm{~T}$ peak field

\begin{tabular}{|c|c|c|c|c|c|c|c|}
\hline \multicolumn{8}{|c|}{ Wiggler \#1 $\quad$ (7-pole) } \\
\hline $\begin{array}{l}\text { Pole Length } \\
\text { (cm) }\end{array}$ & $\mathrm{Nr}$ & $\begin{array}{l}\text { Main Current } \\
\text { (Amp-turns) }\end{array}$ & Main Turns & $\begin{array}{l}\text { Main Width } \\
(\mathrm{cm})\end{array}$ & $\begin{array}{l}\text { Trim Current } \\
\text { (Amp-turns) }\end{array}$ & Trim Turns & $\begin{array}{l}\text { Trim Width } \\
\text { (cm) }\end{array}$ \\
\hline 20 & 5 & $95.00 \mathrm{k}$ & 588 & 2.540 & - & - & - \\
\hline 15 & 2 & $61.07 \mathrm{k}$ & 378 & 1.631 & $0.86 \mathrm{k}$ & 663 & 0.909 \\
\hline \multicolumn{8}{|c|}{$\begin{array}{cc}\text { Wiggler \#2 } & \text { (7-pole) } \\
\end{array}$} \\
\hline 20 & 5 & $95.00 \mathrm{k}$ & 660 & 2.540 & - & - & - \\
\hline 15 & 2 & $57.00 \mathrm{k}$ & 396 & 1.532 & $4.569 \mathrm{k}$ & 684 & 0.965 \\
\hline \multicolumn{8}{|c|}{ Wigglers \#3 - \#6 (8-pole) } \\
\hline 20 & 4 & $95.00 \mathrm{k}$ & 660 & 2.540 & - & $\overline{-}$ & - \\
\hline 15 & 2 & $95.00 \mathrm{k}$ & 660 & 2.540 & - & - & - \\
\hline 10 & 2 & $50.67 \mathrm{k}$ & 352 & 1.354 & $0.993 \mathrm{k}$ & 836 & 1.186 \\
\hline
\end{tabular}

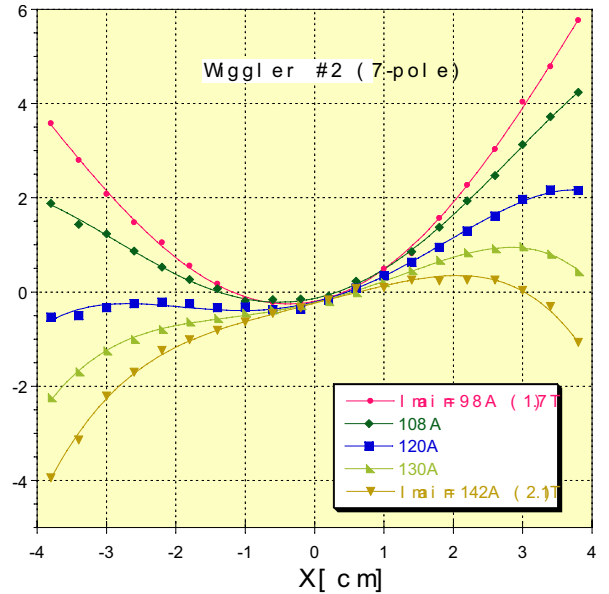

Figure 1: Flip-coil measurements of the integral of the vertical field component for the second of the two 7-pole wigglers as a function of horizontal entrance position in units of Gauss-meters.

determine the value of the second integral. The second integral for the two 7-pole wigglers was measured to be less than $3 \mathrm{G}-\mathrm{m}^{2}$, which corresponds to a displacement of approximately $0.05 \mathrm{~mm}$ for a $1.8 \mathrm{GeV}$ beam. In either design, the dynamic effect on the beam displacement can be reduced to well below $0.1 \mathrm{~mm}$, and so did not influence design decisions.

The flip-coil measurements also quantified the residual integral of the horizontal field component, which has the effect of a skew quadrupole field in the case of a linear horizontal dependence. Figures 3 and 4 show the measurements for the 7- and 8-pole wigglers. Field calculations of geometrical errors within fabrication tolerances showed effects of this magnitude must come from a combination of sources. The combined effect was small enough that a small skew quadrupole magnet sufficed to provide the desired correction during CESR operation with the first wiggler installed in the ring. Space for additional skew quadrupole magnets has been provided for in the final CESR-c design if they turn out to be needed. It is inter-

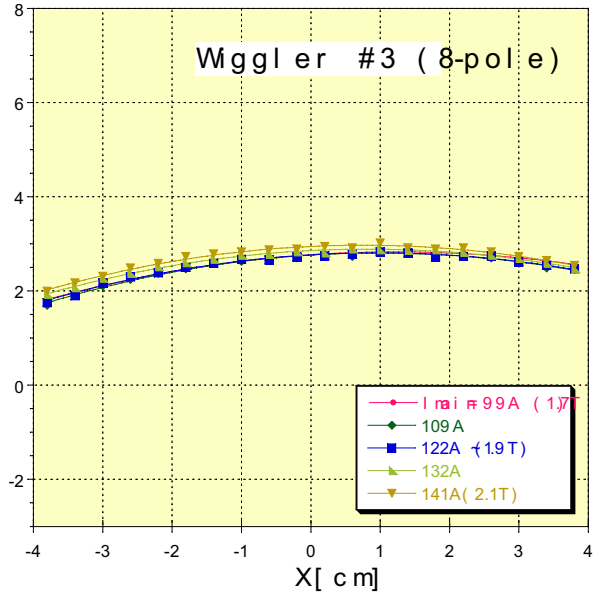

Figure 2: Flip-coil measurements of the integral of the vertical field component for the first of the four 8-pole wigglers as a function of horizontal entrance position in units of Gauss-meters.

esting to note that the effect is much smaller in the 8-pole design, even though the excitation dependence observed in Fig. 3 for the 7-pole design is quite weak.

\section{CALCULATED TRANSFER FUNCTIONS}

The relationship between the field integrals and the transfer functions is complicated by the dynamic effect of the wiggling trajectory, which results in vertical focusing and horizontal kicks which depend on particle energy and entrance position. The horizontal kick for normal incidence in the horizontal symmetry plane as a function of entrance position for $1.8 \mathrm{GeV}$ electrons as calculated with the OPERA tracking algorithm is shown in Fig. 5. The results are consistent with the MERMAID tracking algorithm tracking through the MERMAID-calculated field to an accuracy better than $10 \mu \mathrm{rad}$ over $\pm 3.0 \mathrm{~cm}$. This level of accuracy was also obtained with Runge-Kutta, symplectic integration and Taylor-tracking as described in Ref. [7]. The variation of the horizontal kick with horizontal entrance position is observed to be equal within $10 \mu \mathrm{rad}$ for the 7 - and 


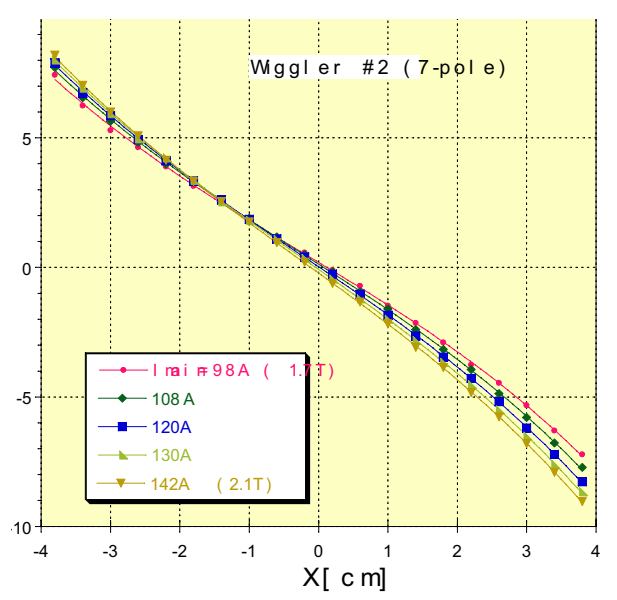

Figure 3: Flip-coil measurements of the integral of the horizontal field component for the second of the two 7-pole wigglers as a function of horizontal entrance position in units of Gauss-meters.

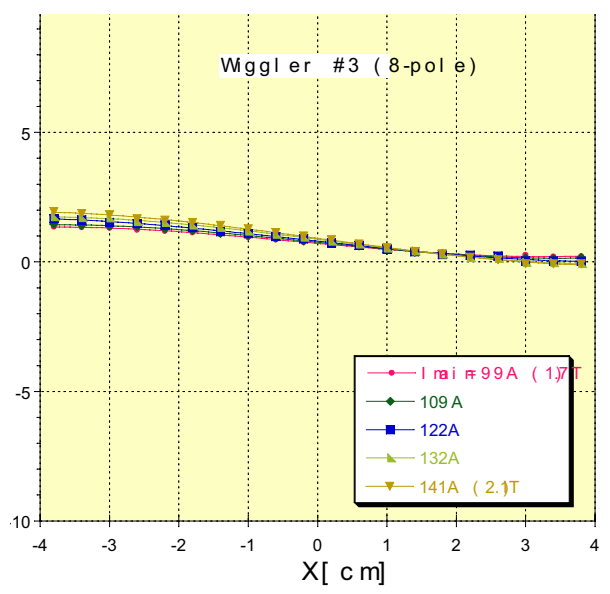

Figure 4: Flip-coil measurements of the integral of the horizontal field component for the first of the four 8-pole wigglers as a function of horizontal entrance position in units of Gauss-meters.

8-pole models. The 8-pole model shows less dependence on excitation, consistent with the flip-coil measurements described above.

The transverse motion of the wiggling trajectory through the longitudinal component of the wiggler field results in a vertical focusing effect. The tracking calculations showed the cubic term in the vertical dependence of the vertical kick to be stronger in the 8-pole design than in the 7-pole design by $2.2 \%$ for a peak field of $1.7 \mathrm{~T}, 4.2 \%$ for $1.9 \mathrm{~T}$ and $5.5 \%$ for $2.1 \mathrm{~T}$.

Beam-based measurements of tune as a function of beam position in the wiggler proved consistent with these calculated transfer functions for peak fields of $1.9 \mathrm{~T}$ and $2.1 \mathrm{~T}[6]$.

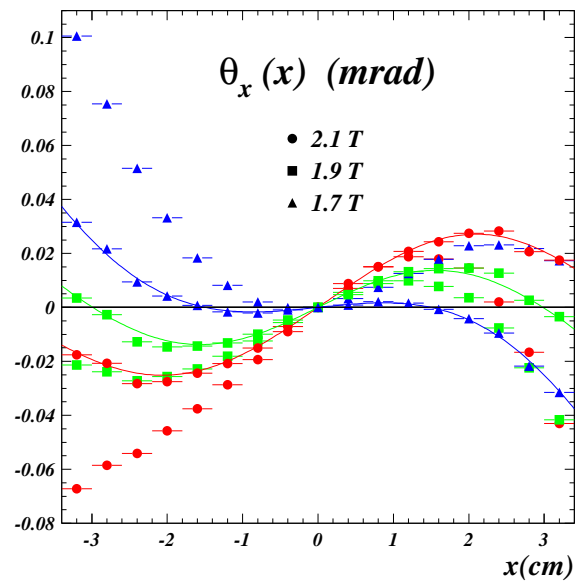

Figure 5: Horizontal kick as a function of horizontal entrance position for $1.8 \mathrm{GeV}$ electrons of perpendicular incidence. Results for peak fields of $1.7 \mathrm{~T}, 1.9 \mathrm{~T}$ and $2.1 \mathrm{~T}$ are shown for the first 7-pole wiggler design and the 8-pole design. Curves connect the points for the 8-pole model.

\section{CONCLUSIONS}

Six superconducting wiggler magnets that meet the design specifications of the CESR-c upgrade have been designed, built and tested. Field maps and transfer functions of sufficient accuracy have been obtained independently with various software packages and computational algorithms. Such construction uniformity was achieved as to allow an 8-pole model to be implemented without necessitating a second trim coil power supply. The 8-pole model exhibits a smaller skew quadrupole component and will allow an increased range of excitation adjustment for tuning purposes during CESR-c beam studies and luminosity optimization. Ten more wigglers of the 8-pole design will be built during the coming year with the goal of installing eight of them in CESR in 2004.

\section{REFERENCES}

[1] D. Rice et al., Parameters for Low-Energy Operation of CESR, proceedings of PAC2001

[2] A. Mikhailichenko, Optimized Wiggler Magnet for CESR, Proceedings of PAC2001, Chicago, IL, June 18-22, 2001

[3] Y. He et al., Design and Operation of the Cryostat for the CESR-c Wiggler Magnets, these proceedings,

[4] D. Rice et al., Production and Testing Considerations for CESRc Wiggler Magnets, these proceedings

[5] A. Temnykh, Vibrating Wire and Flipping-Coil Magnetic Measurements of a CESR-c 7-pole Wiggler Magnet, these proceedings

[6] A. Temnykh, J.A. Crittenden, D. Rice and D. Rubin, Beambased Characterization of a New 7-Pole Super-conducting Wiggler at CESR, these proceedings

[7] D. Sagan, J.A. Crittenden, D. Rubin and E. Forest A Field Model for Wigglers and Undulators, these proceedings

[8] Vector Fields, LTD. http: //www. vectorfields .com 